\title{
Cannabis and its different strains: do they have differential effects on time perception?
}

\author{
Anna Muro, Ramon Cladellas and Judit Castellà \\ Department of Basic, Developmental and Educational Psychology, Universitat Autònoma de \\ Barcelona
}

\section{Author Note}

Anna Muro (D) https://orcid.org/0000-0002-1331-9921

Ramon Cladellas (D) https://orcid.org/0000-0002-0801-8462

Judit Castellà (D https://orcid.org/0000-0002-6094-3516

We have no conflicts of interest to disclose.

Correspondence concerning this article should be addressed to Anna Muro, Department of Basic, Developmental and Educational Psychology, c/ Fortuna s/n Edifici B, Campus UAB, 0819 Bellaterra (Barcelona). Email: anna.muro@uab.cat 


\begin{abstract}
Previous studies show how time perception can be altered by cannabis consumption, but it is not clear yet whether cannabis produces temporal underproductions or overproductions after acute cannabis intoxication. The present study aimed to analyze a sample of 50 regular cannabis users controlling for cannabis strain (sativa, indica, and hybrid) and to compare their scores in a temporal production task before and after consuming cannabis with a control group of 49 non-users. Results showed that cannabis intake leads to overproductions, suggesting that regular users' internal tempo slows down after acute intoxication. However, the analyses of main effects showed that indica users, both at baseline levels and after consuming, reported significant underproductions compared to controls, sativa, and hybrid users, and the cannabis-induced effects had a higher magnitude after smoking in the indica-strain group. Results highlight the relevance of including the type of strain consumed in cannabis studies and they are discussed in terms of short- and long-term alterations in temporal perception under the light of the self-medication theory and the therapeutic uses of cannabis.
\end{abstract}

Keywords: cannabis, temporal perception, regular users, indica, sativa 


\section{Cannabis and its different strains: do they have differential effects on time perception?}

Cannabis is a generic term used to denote the several psychoactive preparations of the plant Cannabis sativa. It is the most prevalent drug used in Europe (UNODC, 2018) and the age for the initiation of its use is around 14 years, situating it as the illegal drug that starts being consumed earlier than any other, and with men showing a higher prevalence of consumption than women (Cuttler et al., 2016; Muro, 2015). It contains more than 70 different cannabinoids, and produces a variety of central effects through its principal active constituent tetrahydrocannabinol (THC), which exerts effects in the brain by binding to a G-protein-coupled receptor known as the CB1 cannabinoid receptor (Atakan et al., 2012; Walsh et al., 2017; Zou \& Kumar, 2018). Cannabinoids increase the activity of dopaminergic neurons, which are known to play a pivotal role in mediating the reinforcing (rewarding) effects and abuse properties of marijuana. Thus, cannabinoids share a final common neuronal action with other major drugs of abuse such as morphine, ethanol, and nicotine in producing facilitation of the mesolimbic dopamine system (Ameri, 1999). Short term effects of cannabis use are significant alterations in consciousness, perception, cognition, affect, or executive functions (Pattij et al., 2008; Sewell et al., 2013; Solowij et al., 2002), while long-term effects have to deal with dependence, mental disorders, or executive impairment and cognitive deterioration (Walsh et al., 2017; WHO, 2016). Nevertheless, self-medication theories suggest that cannabis intake could help individuals modulate specific outcomes of their extreme behaviours and personality. For instance, cannabis would have an inhibitory and relaxing effect for those who express impulsive tendencies and would facilitate the reduction of anxiety for more anxious individuals (Arendt et al., 2007; Casas, 2011; Hall \& Queener, 2007; Muro, 2015). Treatment effectiveness and safety studies have reported sedative, hypnotic, appetite stimulation, 
anticonvulsant, antidepressant, antispasmodic, antiemetic, analgesic, or anti-inflammatory uses associated with this plant (Amar, 2006; Gruber et al., 1996; Robson, 2001; Russo, 2016; Zuardi, 2006).

One of the cognitive mechanisms that has received more attention in the study of cannabis effects is time perception, an important factor for adaptation to the environment and for optimal psychological adjustment (Atakan et al., 2012; Sewell et al., 2013; Wittmann \& Paulus, 2008). Many human behaviours rely on temporal judgments from seconds-to-minutes range that determine human daily decisions based on anticipated duration, such as crossing traffic-lights from amber to red, being on-time at work or follow/dance to the music rhythms. Therefore, disruptions in time perception could have far-reaching functional implications, such as those consistently caused by drugs (Cuttler et al., 2016). It is suggested that cannabis has a significant impact on the activity of the cerebellum, which has one of the highest concentration of cannabinoids binding sites (Zou \& Kumar, 2018). It alters individuals' inner tempo and their ability to accurately estimate time (Correa et al., 2006; Ogden \& Montgomery, 2012) but the specific effects on time perception at long and short term are still debated.

One of the most commonly used tasks in temporal perception literature is production, which consists on asking participants to produce a target temporal interval of a specified duration, with the interval being defined by two responses, or by the duration of a single response such as a button-press (Wearden, 1991). Following the internal clock model (Gibbon et al., 1984) when a production task is performed, any factor that causes a speeding up of our internal tempo, such as arousal or temperature, will imply that more temporal units will be stored faster in working memory when trying to produce a given temporal target, thus leading to underproductions. Therefore, a faster-running internal clock leads to subjective temporal overestimation, as a consequence of the higher amount 
of information in working memory, a phenomenon that has been confirmed by several studies after acute cannabis intoxication (Sewell et al., 2013). By contrast, if the internal clock is slowed down the opposite tends to be observed, that is, participants tend to make longer productions which are due to a faster sense of time passage (underestimation). This opposite effect has also been observed in several cannabis studies (Dornbush \& Kokkevi, 1976; Karniol et al., 1974; Solowij et al., 2002; Vachon et al., 1974) and some authors suggest that the differences found in previous studies might be due to biochemistry or dosage differences in cannabis compounds. Although it is consistently reported that cannabis users frequently report a subjective temporal distortion in the minutes range when they are acutely intoxicated, there is still some controversy on the direction of effects of cannabis on time perception (Atakan et al., 2012; Sewell et al., 2013). Some studies have shown that individuals using cannabis overestimate duration in verbal estimation tasks (Weil et al., 1968; Clark et al., 1970; Pérez-Reyes et al., 1991; Lieving et al., 2006) and show underproductions in time production tasks over a range of 2 seconds to 3 minutes (Dougherty et al., 1994; Hicks et al., 1984; McDonald et al., 2003; Meyer et al., 1971; O’Leary et al., 2003;Pérez-Reyes et al., 1991; Tinklenberg et al., 1976, 1972; Vachon et al., 1974; Sewell et al., 2013; Stone et al., 2010). In rats, it has been also shown that the brain $\mathrm{CB}_{1}$ receptor agonists THC and WIN-55,212 cause time underproduction, as well as in primates (Conrad et al. 1972; Schulze et al., 1988). However, other studies have demonstrated that cannabis consumers tend to underestimate time and overproduce temporal intervals in occasional but also in long-term users (Carlin et al., 1972; Dornbush \& Kokkevi, 1976; Karniol et al., 1974; Solowij et al., 2002; Vachon et al., 1974). Similarly, it has been shown that the $\mathrm{CB}_{1}$ receptor antagonist Rimonabant causes time overproduction in rats (Han \& Robinson 2001), a result that supports the interpretation that different cannabinoids induce different effects in time perception. Several studies report 
that this discrepancy might depend on specific cannabis biochemistry or on the users' profile -occasional, regular or long-term users- that should be considered to control for potentially confounding results.

It is also reported that at baseline, chronic or regular exposure to cannabis does not change basal time perception significantly, suggesting that frequent cannabis smokers experience less temporal distortion than infrequent/non-smokers (Sewell et al., 2013), but this assumption is not consistent with the long-term effects of cannabis in cognitive functions (Walsh et al., 2017; WHO, 2016). In most previous reports, researchers have not reported baseline differences in time perception when compared to controls (Sewell et al., 2013), and those who have reported results have shown a baseline temporal underproduction and overestimation (Bech et al., 1973; Clark et al., 1970; Stone et al., 2010). Other investigators report the opposite: baseline underestimations (Mendhiratta et al., 1978) and overproductions (Hicks et al., 1984; Tinklenberg et al., 1972; Vachon et al., 1974). The reason for this disparity is unclear, and several authors suspect that methodological limitations and some important factors should be better controlled for in these experiments such as the specific cannabis biochemistry (Atakan et al., 2012; Sewell et al., 2013).

Regarding cannabis biochemistry, it is surprising to find that no previous study has included yet the type of cannabis strain as a confounding factor, since formal botanical and pharmacological taxonomy of cannabis has identified three strains (varietals) in the commercial marketplace with different biochemistry (Hillig \& Mahlberg, 2004; McPartland \& Guy, 2017; Pearce, Misouras, \& Irizarry, 2014): 1) Sativa strain has uplifting and more euphoria effects. Sativa plants are said to produce much more $\Delta 9$ tetrahydrocannabinol (THC) than cannabidiol (CBD), and a terpenoid profile that smells "herbal" or "sweet". 2) Indica is more relaxing and shows more sedative effects, and plants 
are said to produce more CBD than Sativa, and a THC-to-CBD ratio closer to 1:1. The terpenoid profile imparts an acrid or "skunky" aroma and finally, 3) Hybrid, which is the result of breeding Sativa and Indica types resulting in intermediate effects. In general, it is considered that sativa plants have a higher ratio of $\mathrm{CBD} / \mathrm{THC}$ and the reverse is seen for indica plants. Whether there is a verifiable difference in the specific effects between Sativa and Indica type strains is still debated (Schwabe \& McGlaughlin, 2019). But it is well known that Sativa is recommended for treating depression, headaches, nausea, and loss of appetite; it causes a stimulating and energizing type of psychoactivity, while Indica is recommended for treating insomnia, anxiety, pain, inflammation, muscle spasms, epilepsy, and glaucoma (Amar, 2006; Gruber et al., 1996; Russo, 2016; Zuardi, 2006).

Nevertheless, to date, the effects of different cannabis strains in temporal perception has not been tested yet, and while these differential effects could be anecdotic for the occasional users, they could be long-term significant for regular users and for patients that are therapeutically prescribed cannabis to alleviate specific medical symptoms (Amar, 2006; Russo, 2016).

Accordingly, this study aims to disentangle how cannabis changes time perception in a Spanish sample of regular consumers, considering cannabis strain (sativa, indica and hybrid) as a co-variable that might potentially explain some of the inconsistent results in previous studies in both baseline levels and in the perception of time after acute cannabis intoxication. The hypotheses are the following:

$\mathrm{H}_{1}$ : Cannabis consumption would alter the perception of time (quantitively and qualitatively) after acute intoxication.

$\mathrm{H}_{2}$ : There would be baseline differences in time perception considering the type of strain consumed among regular cannabis users and compared with the control group. 


\section{Methods}

\section{Design and Procedure}

Given the ethical guidelines regarding experimental designs using drugs, researchers chose a naturalistic study using a quasi-experimental design with a criterion group of regular cannabis smokers and a control group of non-smokers paired by age. Regular cannabis users are defined as smoking at least weekly during a year, but there is a considerable variation in consumption patterns of heavy and light use (Sewell et al., 2013). At any case, regular users are considered to smoke at least two and up to five times a week for one year or more (Chatwin \& Porteous, 2013). The Ethical committee of the university approved the protocol. Participants of the criterion group were asked to collaborate from a randomly chosen Cannabis Social Clubs (CSCs) of the Barcelona area. A Cannabis Social Club, is a club for smokers under legal regulation, organized as non-profit organization, in which cannabis is cultivated and enjoyed collectively, usually for therapeutic purposes or for social engagement (ENCOD 2015; Pardal, 2016). These places differ from Cannabis coffee shops in that those are also operating coffee shops where cannabis is openly sold, while social clubs are usually not selling cannabis, and are only accessible to members. In the criterion group, the experimenter met the participants at the Club, after sending a participation call to all the members of the club by e-mail. E-mails were sent with the participation and permission of the club managers, informing that individual participation in the research call was voluntary and anonymous. There were 77 positive answers to participate in the study, but only 55 met the inclusion criteria: not being diagnosed with any previous or psychiatric disorder, not taking other drugs, and being a regular consumer for more than one year. No standardized questionnaires were administered to collect information regarding psychiatric disorders, other drugs intake, or time of use. Participants filled in a prior informed consent to guarantee the veracity of their data and their voluntary 
and anonymous participation. Each participant met with the experimenter individually at a given time slot, and they sat in a quiet room of the club, with no auditory or social interferences. A total of 50 participants took part in the study and tasks were performed individually. They were asked to wait in the room and not to smoke until researchers explained them the procedure. Researchers explained the study to participants individually: before cannabis consumption, they were asked to perform an adapted production task (a prospective temporal measure which requires the production of an interval of a specified duration, with the interval being defined by two responses). In this case, participants were asked to produce 1 minute using a stopwatch: they had to press a button one time and press again once they thought a minute had passed. The display of the stopwatch was not visible to the participants and their estimation was not reported in order to avoid learning effects that could influence performance in the second phase. They were instructed not to count, which is the simplest and more efficient method of preventing counting in timing tasks (Rattat \& Droit-Volet, 2011). Immediately after they were asked to judge the passage of time of the 1-minute production task, and rate it in a Likert scale ranging from 1 (slow) to 3 (fast). Passage of time judgements are not made in comparison to another event, but instead require participants to decide whether the subjective speed at which they feel time is passing is the same as normal, quicker than normal or slower than normal (Wearden, 2005).

\section{INSERT FIGURE 1 AROUND HERE}

Then, the participants smoked one cigarette of tobacco mixed with their favorite cannabis type at their own pace (one joint). The dosage and amount of cannabis was up to the smoker, but estimations of the average marijuana amount in a joint mixed with tobacco is among $1 / 3$ of the total composition, that is within a $0.3-0.5 \mathrm{~g}$ range (Kilmer and Pacula, 
2009; Ridgeway \& Kilmer, 2016). Since all smokers were associate members of the Cannabis Club, the Club provided the 3 kinds of strains (indica, sativa and hybrid) to perform the experiment, named in plastic bags. Researchers asked to the participants the kind of cannabis that they would smoke according to their consumer history of use before starting the experiment and checked their responses and elections with their purchase histories stored by the Club managers. The Club has the legal permission and obligation to control the different cannabis strains that are sold to the associate members and register all their purchases in an informatic application. So, the cannabis users can buy and smoke their favourite cannabis in the Club, that also controls what type of cannabis is sold and has the purchase history -including dates, frequency, amounts and type of strains sold- of each member.

After 15 minutes, participants were required to perform the two tasks again (production task and passage of time judgement). In the control group, the tasks were performed in a laboratory of the University with university students that also responded to a research call, but in matching conditions: indoors, without auditory and social distractions but no isolated in a cabin. The difference being that they did not smoke and they performed a 15-minutes distracting task instead. Any of the participants in both control and experimental groups received financial or other kind of rewards.

The initial resulting design was a mixed 2 (moment as a within subjects factor) $\mathrm{x} 2$ (group as a between subjects factor) design, with two dependent variables: production and passage of time judgements. The experimental timeline for the experimental group can be seen in Figure 1.

INSERT FIGURE 1 AROUND HERE 


\section{Participants}

A total of 99 participants took part in the study. 50 smokers (21 female and 29 male, mean age $=26.2, \mathrm{SD}=6.2$ ) and a control group of 49 non-smokers recruited ( 27 female and 22 male, mean age $=25.9, \mathrm{SD}=6.1)$. The a priori definition of the regular cannabis-using group, which was designed to identify regular cannabis users, was. Cannabis smokers were members of a cannabis social club of Barcelona city. $20 \%$ of the sample reported smoking only indica type of marihuana, $30 \%$ were sativa type smokers while $50 \%$ smoked hybrid. As a naturalistic design, it was hard to pre-determine the sample size of the experimental group or to find regular users outside cannabis clubs. Accordingly, the final sample size of the cannabis users' group was composed by to those participants that answered the research call of the Cannabis club during two-weeks, and that met the inclusion criteria of being adult aged ( $>18$ years) without current or previous mental health diagnoses. The control group was also a convenience sample of university students that responded to a participation call. Sex and age differences were controlled among both groups, as well as time productions in the pre-tests.

\section{Materials}

An ad-hoc questionnaire was administered to participants that included questions regarding age, gender, education, time of cannabis use (in years), daily amount consumption, and type of cannabis. The qualitative passage of time measure (slow-normal-fast) reported by the participants before and after consumption was also registered in the questionnaire. Finally, a stopwatch was used in order to perform the production task.

\section{Statistical analyses}

Descriptive statistics and Student's t test were obtained for age and pre-experimental scores by gender and experimental group. Means and standard errors as well as Pearson's correlations were also computed between age, time and frequency of cannabis 
consumption, pre and post measures of time estimation in the group of cannabis users. A General Linear Model (GLM) 2 x 2 of repeated measures was performed to contrast the two experimental conditions (scores in pre-post temporal time. A contingency analysis was also performed to contrast the differences among users and non-users in subjective estimation of time passage (slow-normal-fast). To study the potential differential effects in temporal production considering cannabis strain, an Anova was applied contrasting strain intake as group factor (control, indica, sativa or hybrid) as independent variable and time production before and after as dependent variables. Schffe's and nonparametric tests for related samples were performed to contrast time production among the four experimental groups, considering strain intake among the cannabis groups (control, indica, sativa and hybrid). Finally, a contingency analysis was also performed to contrast the differences among users vs non-users in subjective estimation of time passage (slow-normal-fast). The calculations were performed with the SPSS / PC + statistical package (version 19) and the statistical tests were bilateral with variable type I error at $5 \%$.

\section{Results}

Descriptive statistics and correlations among cannabis user's characteristics (age, years of consumption, number of daily cannabis-cigarettes, number of days per week consumption, pre and post measures of quantitative time estimation are reported in Table 1. Student's $t$ test to contrast previous differences considering sex did not show statistically significant differences neither in age $\left(t_{(1,97)}=-.629 ; p=.531\right)$ or in pre-test measures $(t$ $(1,97)=-1.006 ; p=.317)$. Mean age of all participants was $26.06(S D=6.4)$. Time consumption of cannabis for regular users was 3.40 years and daily consumption was of 2.70 cannabis cigarettes per day, 3.08 days per week. As can be also seen in Table 1, age correlated with years of consumption $(r=.358 ; p<.011)$, daily consumption with weekly 
consumption $(r=.316 ; p<.025)$ and pre-scores with post-scores of time production $(r=$ $.674 ; p<.000)$. Since pre-post quantitative temporal productions, which are the variables of interest, did not correlate with the rest of variables, they were not included in further analyses.

\section{INSERT TABLE 1 AROUND HERE \\ INSERT TABLE 2 AROUND HERE}

As reported in Table 2, significant differences were observed in temporal production $(p=.006)$ and in group interaction $(p=.001)$, indicating that the cannabis users-group reported a lower time production than controls during the pre-test while the opposite pattern was observed during the post-test, with a higher time production among the cannabis users, as can be seen in Figure 2. The mean differences were significant when comparing experimental groups at post-measures $\left(F_{(1,97)}=4.26 ; p=.042 ; d=.04\right)$, with cannabis-users showing a post-time production of 4.769 seconds higher than controls. Analyses of time production differences in each group was significant only in the cannabis group $(t=-3.45 ; p=.001 ; d=0.05)$, showing a difference of 5.48 seconds between pre and post measures, while the control group showed a non-significant difference of - 0.428 among pre-post measures of time production.

\section{INSERT TABLE 3 AROUND HERE \\ INSERT FIGURE 2 AROUND HERE}

Further analyses are reported in Table 3, showing mean differences in pre-post temporal production considering separately the main effects of the three different strain- 
intake groups -indica, sativa, hybrid- and control. Although in all three groups cannabis tended to induce overproductions, the impact was much higher in the users who smoke indica and hybrid types (Figure 3). Significant differences were reported at baseline pretask levels between indica and sativa groups $(p=.021)$, between indica and control group in pre $(p=.006)$ and between indica and hybrid, in both pre $(p=.002)$ and post measures $(p$ $=.035)$. Significant differences in post-measures were also observed between control and hybrid $(p=.037)$. Nonparametric tests showed significant differences leading to overproductions when compared to pre-measures after smoking all three cannabis strains, but while indica users still underproduced time in the post-task, sativa $(p=.038)$ and hybrid ( $p=.044$ ) induced overproductions when compared to the pre-measures. Compared to the rest of the groups, indica users showed significant time underproductions in the pre- $(48.50$ seconds) and post-tests (55.20), while hybrid showed the highest overproductions of time at both baseline levels (62.04 seconds) and post-measures (67.64 seconds) (see Table 3).

\section{INSERT FIGURE 3 AROUND HERE}

Regarding qualitative passage of time judgements, chi squared tests did not show significant differences in pre answers, but they showed significant differences in post answers of subjective passage of time considering experimental condition $\left(\chi^{2}=11.441 ; p\right.$ $=.003$ ), showing a higher change in the answers' distribution of cannabis users and reporting pre-post differences, with a decreased number of subjects answering "normal" time passage and an increased number of both "fast" and "slow" answers in the postmeasure (see Table 4).

INSERT TABLE 4 AROUND HERE 


\section{Conclusions and discussion}

The present results are in line with those studies suggesting that cannabis alters individuals' inner tempo and their ability to accurately estimate time (Correa et al., 2006; Ogden \& Montgomery, 2012). More specifically, results suggest that cannabis induce overproduction of time in regular cannabis users, which can be interpreted as temporal underestimation (Carlin et al., 1972; Dornbush \& Kokkevi, 1976; Han \& Robinson, 2001; Karniol et al., 1974; Solowij et al., 2002; Vachon et al., 1974). Thus, the present results using a naturalistic design seem to be in line with the internal clock model (Correa et al., 2006; Gibbon et al., 1984; Ogden \& Montgomery, 2012; O'Leary et al., 2003) suggesting that marijuana might produce changes in the running internal clock that slow down smokers' inner tempo, leading them to a faster sense of time passage, since they press the stopwatch later in the post-production task. However, it has to be noted that the internal clock model assumes a centralized timing mechanism in which the same brain structures and circuitry are involved to determine the duration of many different events. More recent accounts suggest that timing might be distributed, implying more brain areas, that are involved as a function of many variables, such as sensory modality (Mauk \& Buonomano, 2004). Data from drug and lesion studies suggest the implication of a network of brain areas such as the basal ganglia, motor area, prefrontal cortex and posterior parietal cortex (see Atakan et al., 2012 for a review). In this sense, it could be argued that time estimation processes are affected by cannabinoids through compromising the dopaminergic system, and involving varying brain networks. In any case, our study is behavioural and not neurobiological in nature, and therefore strong conclusions cannot be drawn.

Nevertheless, a co-variation has been found when analysing the main effects of the type of strain that have been tested: while all cannabis groups tend to increase the 
production rate after smoking, only indica users, in contrast with hybrid and sativa users, at both baseline and after smoking, underproduce objective time, suggesting a differential effect of indica strain when compared with hybrid and sativa variations. Therefore, the present results would partially support previous research suggesting baseline timeunderproductions but only in cannabis-indica users (Bech et al., 1973; Conrad et al., 1972; Clark et al., 1970; Stone et al., 2010; Schulze et al.,1988).

This remarkable result needs to be qualified: when analyzing the main effects of the three different cannabis strains in the baseline and post measures, it is interesting to see how time productions are different depending on the type of cannabis used, not just at the baseline pre-test measures, but also at the post- measures: On the one hand, the indica users showed significant time underproductions at pre-test baseline levels (48.50 seconds), showing how biased they are at baseline, without having smoked: indica users produce an average of -11.50 seconds under the objective time, while they are much closer to objective time in the post- measure (55.20 seconds), showing that cannabis slowed down their inner tempo by an average of almost 7 seconds, but still underproduced. On the other hand, sativa users showed the most acute time productions among the cannabis groups at baseline levels (60.33 seconds), and also increased their initial productions in the post-task, but to a lesser degree (64.80 seconds); finally, hybrid users showed the highest time productions at both baseline levels (62.04 seconds) and at the post-measures (67.64 seconds) when compared to the rest of the experimental groups. Co-variate results found in the present study regarding baseline differences in time perception among different cannabis-strain uses might suggest that regular intake of cannabis-indica might induce long-term cognitive impairments (Walsh et al., 2017; WHO 2016), and our results cannot support previous findings in which it is reported that it does not (Sewell et al., 2013). This specific result observed for indica-strain is in line with previous animal studies using $\mathrm{CB}_{1}$ 
agonists THC and WIN-55,212 (Conrad et al., 1972; Schulze et al., 1988) and might suggest that the biochemistry of indica strain (with plants producing more CBD than Sativa, and with a THC-to-CBD ratio closer to 1:1) might be more associated with time underproduction. However, the results obtained when analysing global effects in all cannabis users, independent of the strain intake, and the specific main effects observed in sativa and hybrid users, have shown that cannabis intake produces overproduction of temporal intervals after acute intoxication in regular users (Carlin et al., 1972; Dornbush \& Kokkevi, 1976; Karniol et al., 1974; Solowij et al., 2002; Vachon et al., 1974). This might suggest that Sativa, whose plants produce much more THC than CBD and have a higher ratio of $\mathrm{CBD} / \mathrm{THC}$ ) might be associated with time overproduction and the slowing down of the inner clock (Han \& Robinson 2001), a result that supports the interpretation of a differential effect of $\mathrm{CB}_{1}$ agonists in the human brain (Hillig \& Mahlberg, 2004; Pearce, Misouras, \& Irizarry 2014; Schwabe \& McGlaughlin , 2019). Finally, subjective passage of time was also altered among cannabis users, showing significant alterations when comparing pre-post qualitative judgements of cannabis users (Hicks et al., 1984; Mathew et al., 1998; McDonald et al., 2003; Tinklenberg et al., 1972; Sewell et al., 2013). Nevertheless, these alterations do not follow a specific direction and a global alteration is rather observed in qualitative time passage, with judgements showing that all cannabisintoxicated individuals, independently of the strain consumed, change the subjective feeling of normal time passage to both faster and slower time passage.

Accordingly, we can conclude that at short-term or under acute intoxication effects all cannabis users show overproduction of time intervals (temporal underestimation), with all cannabis strains producing a change of more amplitude and magnitude of time intervals. But the main finding of our study suggests that, while acute intoxication of all cannabis types produces overproductions in regular users, baseline group differences might be 
suggesting that Indica strain has a worse long-term impairment of temporal perception related to underproduction. Given the different concentrations of the two main components, with Sativa showing much more THC than CBD and a higher ratio of CBD/THC, and with Indica producing more CBD (Hillig \& Mahlberg, 2004; McPartland \& Guy, 2017), it is suggested that previous inconsistent results in the study of cannabis and temporal perception might be related with the specific and differential biochemistry of the plant variations, rather than the dosage itself (Atakan et al., 2012; Sewell et al., 2013).

Nevertheless, several limitations of the present study should be acknowledged. Firstly, and the most important, the reduced sample size along with the fact that a convenience sample unrepresentative of general population was used, as well as a lack of randomization or lack of a placebo-controlled cross-over study design, limited the generalization of our findings. But it is worth noting that in this naturalistic design, participants did not receive any financial reward for their participation and the recruitment of such specific volunteers (regular cannabis users) made it hard to access to a larger sample size. Secondly, it should be necessary to highlight the fact that this study has only evidenced short-term transitory effects. More research should be carried measuring the duration of these effects and their transfer to daily life in cannabis users compared with other addictions and groups and including follow-up assessments. In any case, this pioneering study encourages the inclusion of cannabis strains -and their associated biochemistry- as a potential co-founding factor that could explain the previous controversy on the effects of cannabis in time perception (Hillig \& Malberg, 2004; Schwabe \& McGlaughlin, 2019; Sewell et al., 2013).

From the self-medication model (Arendt et al., 2007; Casas 2011; Hall and Queener 2007; Muro 2015) and from the perspective of therapeutic cannabis prescription (Amar 2006; Robson 2001; Russo 2016; Zuardi 2006), the present pilot study 
encourages further research analysing strain-differences and suggest that although indicause might produce a higher effect in slowing down inner tempo and thus, in relaxation and in reducing anxiety by overproducing time intervals in the short term, its long-term effects could be worse at impairing temporal perception by fastening the internal clock when regular users are not intoxicated. Thus, it would rather be suggested the use of sativa preparations for safer and more therapeutic uses of cannabis since baseline results of the present study suggest that sativa might impair cognitive functions and long-term mental health (Walsh et al., 2017; WHO, 2016) in a lower degree when compared to regular use of cannabis-indica, but more longitudinal research would be needed to disentangle the differential strain effects observed in this pilot study.

Statement of Ethics and a Disclosure Statement

The authors declare no conflicts of interest. The authors alone are responsible for the content and writing of the paper. The Ethical Committee of the university approved the protocol since the study met the international ethical standards of the Helsinki Declaration (2014).

\section{References}

Ameri, A. (1999). The effects of cannabinoids on the brain. Progress in Neurobiology, 58(4), 315-348.

Amar, M. B. 2006. Cannabinoids in medicine: A review of their therapeutic potential. Journal of Ethnopharmacology 105(1-2): 1-25. 
Arendt, M., Rosenberg, R., Fjordback, L., Brandholdt, J., Foldager, L., Sher, L., \& MunkJørgensen, P. 2007. Testing the self-medication hypothesis of depression and aggression in cannabis-dependent subjects. Psychological medicine 37(7): 935-945.

Atakan, Z., Morrison, P., Bossong, M. G., Martin-Santos, R., \& Crippa, J. A. 2012. The effect of cannabis on perception of time: A critical review. Current Pharmaceutical Design 18(32): 4915-4922.

Bech, P., Rafaelsen, L., \& Rafaelsen, O. J. 1973. Cannabis and alcohol: effects on estimation of time and distance. Psychopharmacologia 32(4): 373-381.

Carlin, A.S., Bakker, C. B., Halpern, L., \& Post, R.D. 1972. Social facilitation of marijuana intoxication: impact of social set and pharmacologica activity. Journal of Abnormal Psychology 80(2): 132-40.

Casas, M. 2011. Self-medication and addictions. In Substance abuse treatment, ed. A.Adan and C. Vilanou, 51-57. Barcelona: Marge Mé dica Books.

Chatwin, C., \& Porteous, D. 2013. Insiders? The experiences and perspectives of longterm, regular cannabis users. Contemporary Drug Problems 40(2): 235-257.

Clark, L. D., Hughes, R., Nakashima, E. N. 1970. Behavioral effects of marihuana: Experimental studies. Archives of General Psychiatry 23(3): 193-198.

Conrad, D. G., Elsmore, T. F., \& Sodetz, F. J. 1972.9 -tetrahydrocannabinol: dose-related effects on timing behavior in chimpanzee. Science 175:547-50.

Correa, A., Lupiañez, J., \& Tudela, P. 2006. The attentional mechanism of temporal orienting: determinants and attributes. Experimental Brain Research 169: 58-68.

Cuttler, C., Mischley, L. K., \& Sexton, M. 2016. Sex differences in cannabis use and effects: a cross-sectional survey of cannabis users. Cannabis and cannabinoid research 1(1): 166-175. 
Dornbush, R. L., \& Kokkevi, A. 1976. Acute effects of cannabis on cognitive, perceptual, and motor performance in chronic hashish users. Annals of the New York Academy of Sciences 282(1): 313-322.

Dougherty, D. M., Cherek, D. R., \& Roache, J. D. 1994. The effects of smoked marijuana on progressive-interval schedule performance in humans. Journal of the experimental analysis of behavior 62(1): 73-87.

ENCOD. 2015. The European cannabis social clubs. Retrieved from https://www.encod.org/info/The-EuropeanCannabis-Social-Clubs.html (accessed 20 April, 2020).

General Assembly of the World Medical Association. 2014. World Medical Association Declaration of Helsinki: ethical principles for medical research involving human subjects. The Journal of the American College of Dentists 81(3): 14.

Gibbon, J., Church, R. M., \& Meck, W. H. 1984. Scalar timing in memory. Annals of the New York Academy of Sciences 423: 52-77.

Gruber, A. J., Pope Jr, H. G., \& Brown, M. E. 1996. Do patients use marijuana as an antidepressant? Depression 4(2): 77-80.

Hall, D. H., \& Queener, J. E. 2007. Self-medication hypothesis of substance use: testing Khantzian's updated theory. Journal of Psychoactive Drugs 39(2): 151-158.

Han, C. J., \& Robinson JK. 2001. Cannabinoid modulation of time estimation in the rat. Behavioral Neuroscience 115:243-6.

Hillig, K. W., \& Mahlberg, P. G. (2004). A chemotaxonomic analysis of cannabinoid variation in Cannabis (Cannabaceae). American journal of botany, 91(6), 966-975.

Hicks, R. E., Gualtieri, C. T., Mayo, J. P., \& Pérez-Reyes, M. 1984. Cannabis, atropine and temporal information processing. Neuropsychobiology 12(4): 229-37. 
Karniol, I. G., Shirakawa, I., Kasinski, N., Pfeferman, A., \& Carlini, E. A. 1974. Cannabidiol interferes with the effects of $\Delta 9$-tetrahydrocannabinol in man. European journal of pharmacology 28(1): 172-177.

Kilmer, B., \& Pacula, R. L. 2009. Estimating the size of the global drug market: A demand-side approach. A Report on Global Illicit Drug Markets, 1998-2007 25(5): $59-78$.

Lieving, L. M., Lane, S. D., Cherek, D. R., \& Tcheremissine, O. V. 2006. Effects of marijuana on temporal discriminations in humans. Behavioural pharmacology 17(2): 173-183.

Lynch, R. C., Vergara, D., Tittes, S., White, K., Schwartz, C. J., Gibbs, M. J., ... \& Kane, N. C. 2016. Genomic and chemical diversity in Cannabis. Critical Reviews in Plant Sciences 35(5-6): 349-363.

Mathew, R. J., Wilson, W. H., Turkington, T. G., \& Coleman, R. E. 1998. Cerebellar activity and disturbed time sense after THC. Brain Research 797(2):183-189.

Mauk M. D., Buonomano, D.V. 2004. The neural basis of temporal processing. Annual Review Neuroscience 27:307-40. doi: 10.1146/annurev.neuro.27.070203.144247. PMID: 15217335.

McDonald, J., Schleifer, L., Richards, J. B., \& de Wit, H. 2003. Effects of THC on behavioral measures of impulsivity in humans. Neuropsychopharmacology 28(7): 1356-1365.

McPartland, J. M., \& Guy, G. W. (2017). Models of cannabis taxonomy, cultural bias, and conflicts between scientific and vernacular names. The botanical review, 83(4), 327381.

Mendhiratta, S. S., Wig, N. N., \& Verma, S. K. 1978. Some psychological correlates of long-term heavy cannabis users. The British Journal of Psychiatry 132(5): 482-486. 
Meyer, R.E., Pillard R.C., Shapiro L. M., Mirin, S.M. 1971. Administration of marijuana to heavy and casual marijuana users. American Journal of Psychiatry 128:198-203.

Muro i Rodríguez, A. 2015. Age, sex and personality in early cannabis use. European Psychiatry 30(4): 469-473.

Ogden, R. \& Montgomery, C. 2012. High time. Psychologist 25(8).

O'Leary, D., Block, R. I., Turner, B. M., Koeppel, J., Magnotta, V. A., Ponto. L. B., Watkins, G. L, Hichwa, R. D., \& Andreasen, N. C. 2003. Marijuana alters the human cerebellar clock. Neuroreport, 14(8):1145-1151.

doi:10.1097/01.wnr.0000075420.59944.20

Pardal, M. 2016. Cannabis Social Clubs through the lens of the drug user movement. Tijdschrift over Cultuur and Criminaliteit 6(2): 47-58.

Pattij, T., Wiskerke, J., Schoffelmeer, A.N. 2008. Cannabinoid modulation of executive functions. European Journal of Pharmacolog, 585(2-3): 458-63.

Pearce, D. D., Mitsouras, K., \& Irizarry, K. J. 2014. Discriminating the effects of Cannabis sativa and Cannabis indica: a web survey of medical cannabis users. The journal of alternative and complementary medicine 20(10): 787-791.

Pérez-Reyes, M., White, W. R., McDonald, S. A., Hicks, R. E., Jefcoat, A. R., \& Cook, C. E. 1991. The pharmacologic effects of daily marijuana smoking in humans. Pharmacology, Biochemistry, and Behavior 4: 691-694. doi: 10.1016/00913057(91)90384-e

Rattat, A., Droit-Volet, S. 2011. What is the best and easiest method of preventing counting in different temporal tasks? Behavioural Research Methods 44(1): 67-90. doi: 10.3758/s13428-011-0135-3. PMID: 21789731. 
Ridgeway, G., \& Kilmer, B. 2016. Bayesian inference for the distribution of grams of marijuana in a joint. Drug and alcohol dependence 165: 175-180.

Robson, P. 2001. Therapeutic aspects of cannabis and cannabinoids. The British Journal of Psychiatry 178(2): 107-115.

Russo, E. B. 2016. Current therapeutic cannabis controversies and clinical trial design issues. Frontiers in pharmacology 7:309.

Sawler, J., Stout, J. M., Gardner, K. M., Hudson, D., Vidmar, J., Butler, L., ... \& Myles, S. 2015. The genetic structure of marijuana and hemp. PloS one 10(8).

Schulze, G.E., McMillan, D. E., Bailey, J. R., Scallet, A., Ali, S. F., Slikker, W., Paule, M. G. 1988. Acute effects of delta-9-tetrahydrocannabinol in rhesus monkeys as measured by performance in a battery of complex operant tests. Journal of Pharmacology and Experimental Therapeutics 245(1):178-86.

Schwabe, A. L., \& McGlaughlin, M. E. 2019. Genetic tools weed out misconceptions of strain reliability in Cannabis sativa: Implications for a budding industry. Journal of Cannabis Research 1(1): 3 .

Sewell, R. A., Schnakenberg, A., Elander, J., Radhakrishnan, R., Williams, A., Skosnik, P.D., Pittman, B., Ranganathan, M., and D'Souza, D. C. 2013. Acute effects of THC on time perception in frequent and infrequent cannabis users. Psychopharmacology 226(2): 401-413. doi: 10.1007/s00213-012-2915-6. Epub 2012 Nov 24.

Solowij, N., Stephens, R.S., Roffman, R.A., Babor, T., Kadden, R., Miller, M., Christiansen, K., McRee, B., \& Vendetti, J. 2002. Cognitive functioning of long-term heavy cannabis users seeking treatment. JAMA 287(9): 1123-31.

doi:10.1001/jama.287.9.1123 
Stone, J. M., Morrison, P. D., Nottage, J., Bhattacharyya, S., Feilding, A., \& McGuire, P. K. 2010. Delta-9-tetrahydrocannabinol disruption of time perception and of self-timed actions. Pharmacopsychiatry 43(06): 236-237.

Tinklenberg, J. R., Kopell, B. S., Melges, F. T., \& Hollister, L. E. 1972. Marihuana and alcohol, time production and memory functions. Archives of General Psychiatry 27(6): 812-815. doi:10.1001/archpsyc.1972.01750300074013

Tinklenberg, J.R., Roth, W.T., \& Kopell, B.S. 1976. Marijuana and ethanol: Differential effects on time perception, heart rate, and subjective response. Psychopharmacology 49: 275-279.

United Nations Office on Drugs and Crime (UNODC). 2018. World Drug Report 2018 Global Overview of Drug Demand and Supply. Retrieved from https://www.unodc.org/wdr2018/prelaunch/WDR18_ExSum_Spanish.pdf

Vachon, L., Sulkowski, A., \& Rich, E. 1974. Marihuana effects on learning, attention and time estimation. Psychopharmacologia 39(1):1-11. doi: 10.1007/bf00421453

Walsh, Z., Gonzalez, R., Crosby, K., Thiessen, M. S., Carroll, C., \& Bonn-Miller, M. O. 2017. Medical cannabis and mental health: A guided systematic review. Clinical psychology review 51: 15-29.

Wearden, J. H. 1991. Do humans possess an internal clock with scalar timing properties?. Learning and motivation 22(1-2): 59-83. doi: 10.1016/0023-9690(91)90017-3

Wearden, J. H. 2005. The wrong tree: time perception and time experience in the elderly. In Duncan, J., Phillips, L., McLeod, P. (Eds). Measuring the Mind: Speed, control, and age. Oxford University Press. doi:10.1093/acprof:oso/9780198566427.003.0006

Weil, A. T., Zinberg, N. E., \& Nelsen, J. M. 1968. Clinical and psychological effects of marihuana in man. Science 162(3859): 1234-124 
Wittmann, M., \& Paulus, M. P. 2008. Decision making, impulsivity and time perception. Trends in cognitive sciences 12(1): 7-12.

World Health Organization. 2016. Health and Social Effects of Nonmedical Cannabis Use. World Health Organization. Retrieved from https://apps.who.int/iris/bitstream/handle/10665/251056/9789241510240eng.pdf;jsessionid=80F338A25436D416C949EF7F06A9A054? sequence $=1$

Zou, S., \& Kumar, U. (2018). Cannabinoid receptors and the endocannabinoid system: signaling and function in the central nervous system. International journal of molecular sciences, 19(3), 833. doi:10.3390/ijms 19030833

Zuardi, A. W. 2006. History of cannabis as a medicine: a review. Brazilian Journal of Psychiatry 28(2): 153-157. 
Table 1. Mean, standard deviation, maximum, minimum and correlations among age, years of consumption, number of daily cannabis use (number of cigarettes), number of days per weekly consumption, pre and post measures of quantitative time estimation.

\begin{tabular}{lllllll}
\hline & 1 & 2 & 3 & 4 & 5 & 6 \\
\hline 1. Age & & & & & & \\
2. Years of use & $.358^{*}$ & & & & & \\
3. Daily intake & .137 & .177 & & & & \\
4. Week days & .038 & -.168 & $.316^{*}$ & & & \\
5. Pre-test & -.217 & .023 & .087 & -.013 & & \\
6. Post-test & .116 & -.022 & -.004 & .129 & $.674^{* *}$ & \\
\hline Mean & 26.06 & 3.40 & 3.08 & 2.70 & 58.82 & 64.30 \\
\hline Standard deviation & 6.40 & 1.05 & 1.47 & 0.58 & 12.43 & 14.84 \\
\hline Maximum & 59.00 & 5.00 & 5.00 & 3.00 & 98.00 & 140.00 \\
\hline Minimum & 19.00 & 1.00 & 1.00 & 1.00 & 24.00 & 41.00 \\
\hline Note: * $\mathrm{p}<0.05 ; * * \mathrm{p}<0.01 ;$ Pre-test: one minute estimation in seconds; post-test: one minute estimation in seconds.
\end{tabular}

Table 2. Descriptive statistics (mean, standard error of the means, t-test, eta squared and observed power) of time estimation by experimental groups (control and experimental).

\begin{tabular}{|c|c|c|c|c|}
\hline \multirow[b]{2}{*}{ Experimental group } & \multicolumn{2}{|c|}{ Time production } & \multirow[b]{2}{*}{$t$} & \multirow[b]{2}{*}{$P$} \\
\hline & $\begin{array}{c}\text { Pre } \\
M \pm S E\end{array}$ & $\begin{array}{c}\text { Post } \\
M \pm S E\end{array}$ & & \\
\hline $\begin{array}{l}\text { Controls } \\
(n=49)\end{array}$ & $59.96 \pm 1.39$ & $59.53 \pm 1.64$ & .52 & .606 \\
\hline \multirow[t]{2}{*}{$\begin{array}{l}\text { Cannabis users } \\
(n=50)\end{array}$} & $58.82 \pm 1.38$ & $64.30 \pm 1.63$ & -3.45 & .001 \\
\hline & & & $F$ & $p$ \\
\hline Time production & & & 7.88 & $.006^{*}$ \\
\hline Experimental group & & & .87 & .353 \\
\hline Interaction & & & 10.78 & $.001 *$ \\
\hline
\end{tabular}

Note: $* \mathrm{p}<.05, * * \mathrm{p}<.01$ 
Table 3. Descriptive statistics (mean, standard error of the means, ANOVA) of time estimation by experimental groups considering type of strain use $\mathrm{Z}$ and $\mathrm{p}$

\section{Type of strain groups}

\begin{tabular}{lcccccc}
\hline $\begin{array}{l}\text { Time } \\
\text { production }\end{array}$ & $\begin{array}{c}\text { Indica } \\
(\mathrm{n}=10)\end{array}$ & $\begin{array}{c}\text { Sativa } \\
(\mathrm{n}=15)\end{array}$ & $\begin{array}{c}\text { Hybrid } \\
(\mathrm{n}=25)\end{array}$ & $\begin{array}{c}\text { Control } \\
(\mathrm{n}=49)\end{array}$ & $F$ & $p$ \\
\hline Pre & $48.50 \pm 4.04$ & $60.33 \pm 1.69$ & $62.04 \pm 2.63$ & $59.96 \pm .84$ & 5.61 & $.001^{*}$ \\
Post & $55.20 \pm 2.84$ & $64.80 \pm 2.35$ & $67.64 \pm 3.61$ & $59.53 \pm .93$ & 4.53 & $.005^{*}$ \\
\hline$Z$ & -1.69 & -2.08 & -2.02 & -1.22 & & \\
$p$ & .091 & $.038^{*}$ & $.044^{*}$ & .224 & & \\
$d$ & & .56 & .35 & & & \\
\hline Notes: ${ }^{*}<.05$. & & & & & &
\end{tabular}

Table 4. Pre-post measures in subjective time estimation by experimental conditions.

\begin{tabular}{cccccc} 
& & \multicolumn{5}{c}{ Experimental group } \\
\cline { 3 - 6 } & & \multicolumn{3}{c}{ Control } & Cannabis \\
\cline { 3 - 6 } & \multirow{2}{*}{ SLOW } & 13 & Post & Pre & Post \\
\hline \multirow{2}{*}{$\begin{array}{c}\text { Subjective Time } \\
\text { estimation }\end{array}$} & \multirow{2}{*}{ NORMAL } & 33 & 6 & 7 & 16 \\
& & $(33,3 \%)$ & $(36,4 \%)$ & $(3,1 \%)$ & $16,2 \%$ \\
\cline { 2 - 6 } & \multirow{2}{*}{ FAST } & 3 & 7 & 35 & 20 \\
& & $(3,0 \%)$ & $(7.1 \%)$ & $8,1 \%$ & $(20,2 \%)$ \\
\hline
\end{tabular}

